\title{
GI-MS45-P03 | EXTENDING NXMX METADATA TO FACILITATE DATA SHARING
}

Bernstein, Herbert J. (Ronin Institute for Independent Scholarship, Upton, NY, USA)

It is increasingly important to be able to combine data from multiple experiments by multiple experimenters at multiple facilities. For synchrotron $\mathrm{MX}$ data there are two major standards for diffraction images: the Crystallographic Binary Format (CBF/imgCIF) [1] adopted for the Dectris Pilatus in 2007 and the NeXus/HDF5 NXmx applications definitions [2]. The High Data Rate Crystallography (HDRMX) group has been working to extend and harmonize the necessary additions to $\mathrm{NXmx}$ and $\mathrm{CBF}$ to facilitate data sharing.

[1] H. J. Bernstein, A. P. Hammersley. "Specification of the Crystallographic Binary File (CBF/imgCIF)." International Tables for Crystallography (2006).

[2] H. J. Bernstein. "Software for processing high-data-rate MX in CIF and NeXus/HDF5." Foundations of Crystallography 70 (2017): C1363. 